Journal of Medical and Biomedical Sciences (2013) 2(4): 35-41

C UDS Publishers Limited All Right Reserved 2026-6294

ORIGINAL ARTICLE

doi: http://dx.doi.org/10.4314/jmbs.v2i4.5

\title{
Co-existence of malaria and urinary tract infection among children under five: A cross-sectional study of the Assin-South Municipality, Ghana
}

\author{
R.K.D. Ephraim ${ }^{1}$, M.A. Nyame ${ }^{1}$, S.A. Sakyi ${ }^{2}$, E.O. Antoh ${ }^{1}$ and D.L. Simpong ${ }^{1}$ \\ ${ }^{1}$ Medical Laboratory Division, Department of Laboratory Technology, University of Cape Coast; ${ }^{2}$ Department of Molecular Medicine, \\ School of Medical Sciences, College of Health Sciences, KNUST, Ghana
}

\begin{abstract}
Across tropical Africa, febrile children are treated for malaria either with or without confirmation thus resulting in failure to diagnose and treat other co-morbidities like urinary tract infections (UTI) and upper respiratory tract infection (URTI) that may coexist with malaria. This crosssectional study examined coexisting malaria with UTI and further assessed the antimicrobial susceptibility pattern of the isolated organisms among children aged $<5$ years presenting with fever. Between December 2012 and May 2013, 284 children were recruited from the Saint Francis Xavier Hospital, in the Central Region of Ghana through purposive sampling. Thick and thin blood films were used for the diagnosis of malaria and urine samples were collected in sterile, wide-mouthed, leak proof containers for culture and sensitivity. Organisms isolated were identified and tested for their antimicrobial sensitivity patterns using the Kirby-Bauer disc diffusion method. Prevalence of malaria with coexisting UTI was $15.8 \%$ with majority $(58.0 \%)$ of the participants being female. Age was significantly $(\mathrm{p}=\mathbf{0 . 0 2 5})$ associated with malaria and UTI co-infection with the highest prevalence of co-infection (35.6\%) recorded amongst the 13-24 months age group; gender was not associated with co-infection $(p>0.05)$. Malaria parasitaemia $(1+$ to $3+)$ was significantly $(p=0.001)$ associated with bacteriuria. Staphylococcus aureus $(30.3 \%)$, Escherichia coli $(20.4 \%)$ and Proteus species $(5.3 \%)$ were isolated and these isolates were highly susceptible to Gentamicin (GEN), Ciprofloxacin (CIP) and Nitrofurantoin (NIT) but were resistant to ampicillin (AMP). Staphylococcus aureus was the predominant cause of the UTI and the isolates were highly resistant to ampicillin but susceptible to gentamicin, ciprofloxacin and nitrofurantoin.

Journal of Medical and Biomedical Sciences (2013) 2(4), 35-41
\end{abstract}

Keywords: Malaria, UTI, antibiotic sensitivity pattern, parasitaemia, bacteriuria, fever

\section{INTRODUCTION}

Malaria has been a global public health problem accounting for approximately $30 \%$ of outpatient and $50 \%$ of in-patient hospital admissions among children under the age of five years especially in subSaharan Africa (Akpede and Skyes, 1992). For decades, majority of African children under the age of 5 years have been presumptively treated for malaria once they present with fever (Rougemont et al., 1991; Kallander et al., 2004; English et al., 2009).

Correspondence: Richard K.D. Ephraim; Medical Laboratory Division, Department of Laboratory Technology, University of Cape Coast, Cape Coast, Ghana; E-mail: rephraim@ucc.edu.gh
Public health studies have established that more than $50 \%$ of African children who present with fever to healthcare centres do not have malaria infection (Gething et al., 2010). In countries where presumptive diagnosis has become a standard practice, various challenges in treatment and management arise as the origin of febrile illness may be due to other causes such as bacterial and viral infections and not exclusively malaria (Kallander et al., 2004).

Co-existence of urinary tract infection (UTI) with these febrile illnesses especially malaria has been reported by several authors across the African continent (Akpede and Skyes, 1992; Okwara et al., 
Malaria and UTI among children

Ephraim et al.,

2004; Okunola et al., 2012). In Nigeria, 9\% of children under the age of five years had malaria coexisting with UTI and this occurred in areas where the diagnosis of malaria were solely based on clinical presentation due to inadequate laboratory facilities (Okunola et al., 2012). In recent times when most healthcare facilities have the capacity to diagnose malaria in well-equipped laboratories, some clinicians still seek to presumptively diagnose children less than five years who present with fever as having malaria (Reyburn et al., 2006; English et al., 2009). This has led to calls for patients to be pre-tested for the presence of malaria parasites before treatment is initiated (Rougemont et al., 1991; Reyburn et al., 2006; English et al., 2009). The purpose of this study therefore, was to establish the prevalence of malaria coexisting with UTI among children $<5$ years in the Assin South municipality and to further assess the antimicrobial susceptibility pattern of the isolated organisms.

\section{MATERIALS AND METHODS}

\section{Study design and participants selection}

This descriptive cross-sectional study was conducted from December 2012 to May 2013 at the Saint Francis Xavier Hospital, in the Assin Central Municipality of the Central Region. Children aged $<5$ years who reported to the health facility with signs and symptoms of malaria during the study period were eligible for this study. Three hundred and five (305) of these participants with clinically diagnosed and/or laboratory confirmed malaria were recruited for the study using purposive sampling. A total of 284 children were finally enrolled due to the inability of 21 participants to provide urine samples and other vital data. Informed consent was obtained from parents or guardians of all the participants prior to enrolment. The study was approved by the Institutional Review Board of the University of Cape Coast (IRB/UCC) and the Ethics Committee of the Saint Francis Xavier Hospital, Assin-Fosu.

\section{Inclusion criteria}

Children aged $<5$ years with clinically diagnosed (WHO, 2000) and microscopically demonstrated malaria were considered eligible to participate in the study. Participants with axillary temperature at least $38.0^{\circ} \mathrm{C}$ and not having received antimalarial and antibiotic treatments prior to enrolment were included in the study.

\section{Exclusion criteria}

The following were excluded from the study: children exposed to antibiotics within 14 days prior to the study, children with enteric fever, hepatitis, acute gastroenteritis, meningitis, viral infections (mumps, measles), pharyngo-tonsillitis, bronchopneumonia, recurrent urinary tract infections and otitis media. Similarly, children with catheterization done within three days before the study and those with immunosuppression due to sickle cell disease, malnutrition and human immune virus/acquired immunodeficiency syndrome (HIV/AIDS) were not selected.

\section{Malaria diagnosis}

Thick and thin Giemsa-stained blood smears were examined for the presence of Plasmodium falciparum parasites using standard techniques (WHO, 1991a). The parasite count and identification were determined semi-quantitatively (WHO, 1991b).

\section{Collection of urine samples for Urine Culture and Sensitivity $(\mathrm{C} / \mathrm{S})$}

A sterile, dry, wide necked, leak proof screw capped container was used to collect midstream urine (MSU) specimen for urinalysis, microscopy, culture and sensitivity. The specimen were refrigerated immediately upon getting to the laboratory or cultured within 2 hours.

A standard calibrated loop was used to fetch a loopful $(0.002 \mathrm{ml})$ of well mixed urine sample and inoculated on Cysteine Lactose ElectrolyteDeficient (CLED) agar. This was incubated aerobically at $37^{\circ} \mathrm{C}$ for $18-24$ hours in an incubator (IPF 400 Precision, Memmert, Germany).

\section{Identification and Counting of Bacterial Iso- lates \\ Bacterial colonies were identified based on colonial morphology (color, growth size, and growth pat- tern). Standard biochemical tests: citrate, urease,}


Malaria and UTI among children

Ephraim et al.,

indole, catalase, and coagulase tests were used for further identification of isolates. Bacterial count was estimated from the product of the loop volume and the colony count on CLED. Bacterial counts $>1 \times 10^{5}$ $\mathrm{CFU} / \mathrm{ml}$ was considered significant whilst bacterial counts between $1 \times 10^{4}-10^{5} \mathrm{CFU} / \mathrm{ml}$ was considered doubtfully significant. Bacterial count $<1 \times 10^{4}$ $\mathrm{CFU} / \mathrm{ml}$ was considered insignificant (Harding et al., 2002).

\section{Antimicrobial Susceptibility Test (AST)}

The Kirby-Bauer disc diffusion method (Bauer et al., 1966) was used to determine the susceptibility of the isolates to selected antimicrobial agents. Antibioticimpregnated paper discs (Medical wire and Equipment Co. Ltd., PotleyCorsham, England) containing the following antibiotics: Nalidixic acid (NAL, 30 $\mu \mathrm{g})$, Gentamicin (GEN, $10 \mu \mathrm{g}$ ), Tetracycline (TET, $30 \mu \mathrm{g})$, Nitrofurantoin (NIT, $15 \mu \mathrm{g}$ ), Cotrimoxazole (COT, $25 \mu \mathrm{g}$ ), Ampicillin (AMP, $10 \mu \mathrm{g}$ ), Cefuroxime (CRX, $30 \mu \mathrm{g}$ ) and Pipemedic acid (PPA, $30 \mu \mathrm{g}$ ) were utilized for susceptibility tests.

A straight wire loop was used to emulsify fresh isolates of pure colonies in peptone water and the turbidity adjusted to $0.5 \mathrm{McF}$ arland's standard. Using a sterile cotton swab, a portion of the emulsified suspension was seeded on Mueller-Hinton agar plate in a three dimensional streak fashion.. Antibiotic discs for urine pathogens was then placed on the plated agar within 15 minutes of seeding and then incubated at $37^{\circ} \mathrm{C}$ overnight $(18-24$ hours). A caliper was used to determine the zone of inhibition in millimeters which was then compared to a standard chart to determine susceptibility categorized as sensitive or resistant as previously described by Tagoe and Desbordes (2012). A Gram negative-organism Escherichia coli (NCTC 10418) and Staphylococcus aureus [National collection of type cultures (NCTC) 6571], a Gram-positive organism, were used as controls.

\section{Statistical analysis}

GraphPad Prism version 5.00 for windows was used for statistical analysis (GraphPad software, San Diego California USA, www.graphpad.com). The results were expressed as Means \pm SD. Unpaired t-test was used to compare mean values of continuous varia- bles and $\chi^{2}$ was used to compare discontinuous variables and P-values $<0.05$ were regarded as significant.

\section{RESULTS}

As shown in Table 1, the mean age of study participants was $35.7 \pm 15.7$ months. The mean age of males and females was not significantly different $(\mathrm{P}=0.723)$. More females fell within the age groups of 13-24 (14.8\%), 25-26 (13.0\%) and 37-48 (10.9\%) months respectively compared to males of the same age $(9.9 \%, 12.3 \%$ and $8.5 \%)$. The number of males and females within the various age groups did not differ statistically $(\mathrm{P}=0.326)$. Female participants with parasite densities above " $1+$ " (" $2+$ ", " $3+$ " and "4+") were more than males $(\mathrm{P}=0.402)$. There were more males with a "1+" parasite density than females $(\mathrm{P}=0.402)$. More males than females had gram positive cocci $(15.5 \%)$ whereas more females $(14.8 \%)$ than males had gram negative rods. Although Staphylococcus aureus was the predominant isolate among the study participants, it was more among the males $(15.5 \%)$ than females $(14.8 \%)$ with more strains of Escherichia coli and Proteus species among the females (10.8\% and 3.5\% respectively). There was no significant difference in gram reaction $(\mathrm{P}=0.654)$ and types of isolates $(\mathrm{P}=0.640)$ between males and females (Table 1).

Generally there were more children with bacteriuria than those without within all the age groups with the exception of those from 37-48 months where there were more participants without bacteriuria $(23.2 \%)$ than those with bacteriuria $(16.4 \%)$. There was no significant difference between the ages of participants with and without bacteriuria $(\mathrm{P}=0.444)$ (Table 2).

Though bacteriuria was more prevalent in males compared to females, gender was not associated with bacteriuria $(\mathrm{P}=0.973)$. Bacteriuria was lower among those with detected parasitaemia of all grades and numbers of children with bacteriuria reduced significantly $(\mathrm{P}=0.001)$ with increasing parasite burden (Table 2). 
Malaria and UTI among children

Ephraim et al.,

Table 1: General characteristics of study participants stratified by gender

\begin{tabular}{lcccc}
\hline Variables & Male $\mathbf{n}=\mathbf{1 3 6}$ & Female $\mathbf{n = 1 4 8}$ & Total $\mathbf{n}=\mathbf{2 8 4}$ & P value \\
\hline Mean Age & $36.02 \pm 16.5$ & $35.36 \pm 14.9$ & $35.7 \pm 15.7$ & 0.723 \\
Age range (months) & & & & \\
$\leq 12$ & $23(8.1)$ & $18(6.3)$ & $41(14.4)$ & 0.326 \\
$13-24$ & $28(9.9)$ & $42(14.8)$ & $70(24.6)$ & \\
$25-26$ & $35(12.3)$ & $37(13.0)$ & $72(25.4)$ & \\
$37-48$ & $24(8.5)$ & $31(10.9)$ & $55(19.4)$ & \\
$49-60$ & $26(9.2)$ & $20(7.0)$ & $46(16.2)$ & \\
Parasite density & & & & \\
No mps & $75(26.4)$ & $84(29.6)$ & $159(56.0)$ & 0.402 \\
$1+$ & $39(13.7)$ & $32(11.3)$ & $71(25.0)$ & \\
$2+$ & $16(5.6)$ & $23(8.1)$ & $39(13.7)$ & \\
$3+$ & $6(2.1)$ & $7(2.5)$ & $13(4.6)$ & \\
$4+$ & $0(0.0)$ & $2(0.7)$ & $2(0.7)$ & \\
Gram reaction & & & & \\
None & $60(21.1)$ & $65(22.9)$ & $125(44.0)$ & 0.654 \\
GPC & $44(15.5)$ & $42(14.8)$ & $86(30.3)$ & \\
GNR & $32(11.3)$ & $41(14.4)$ & $73(25.7)$ & \\
Isolated organism & & & & \\
None & $60(21.1)$ & $65(22.9)$ & $125(44.0)$ & 0.640 \\
Staphylococcus aureus & $44(15.5)$ & $42(14.8)$ & $86(30.3)$ & \\
Escherichia coli & $27(9.5)$ & $31(10.8)$ & $58(20.4)$ & \\
Proteus spp. & $5(1.8)$ & $10(3.5)$ & $15(5.3)$ & \\
\hline
\end{tabular}

GR: Gram reaction; GPC: Gram positive cocci; GNR: Gram negative rod; mps: malaria parasites

Table 2: Characteristics of study participants based on the present or absent of bacteriuria

\begin{tabular}{lccc}
\hline Variable & $\begin{array}{c}\text { Present } \\
\mathbf{n = 1 5 9}\end{array}$ & $\begin{array}{c}\text { Absent } \\
\mathbf{n = 1 2 5}\end{array}$ & P-value \\
\hline $\begin{array}{l}\text { Age } \\
\text { (months) }\end{array}$ & & & \\
$\leq 12$ & $21(13.2)$ & $20(16.0)$ & 0.444 \\
$13-24$ & $43(27.0)$ & $27(21.6)$ & \\
$25-26$ & $44(28.0)$ & $28(22.4)$ & \\
$37-48$ & $26(16.4)$ & $29(23.2)$ & \\
$49-60$ & $25(15.7)$ & $21(16.8)$ & \\
Gender & & & \\
Male & $76(47.8)$ & $60(48.0)$ & 0.973 \\
Female & $83(52.2)$ & $65(52.0)$ & \\
Parasite & & & \\
density & & & \\
No mps & $114(71.6)$ & $45(36.0)$ & 0.001 \\
1+ & $30(19.0)$ & $41(32.8)$ & \\
$2+$ & $12(7.5)$ & $27(21.6)$ & \\
$3+$ & $3(2.0)$ & $10(8.0)$ & \\
$4+$ & $0(0.0)$ & $2(1.6)$ & \\
\hline
\end{tabular}

mps: malaria parasites
The degree of parasitaemia was significantly associated with number of bacteria isolated $(\mathrm{P}=0.001)$. The highest frequency of bacteriuria was among those without parasitaemia $(71.7 \%)$; the frequency of bacteria declined as the level of parasitaemia increased. For parasite densities of $1+, 2+$ and $3+$ respectively, S. aureus $(39.6 \%, 19.7 \%, 15.4 \%)$ was isolated more than E. coli $(25.2 \%, 16.9,12.8)$ and Proteus spp. $(6.9 \%, 5.6 \%, 0.0 \%)$. In participants with

Table 3: Relationship between parasite density and bacterial isolates of subjects

\begin{tabular}{lccccc}
\hline $\begin{array}{l}\text { Para- } \\
\text { site } \\
\text { density }\end{array}$ & Total & $\begin{array}{c}\text { Staph. } \\
\text { aureus }\end{array}$ & E. coli & $\begin{array}{c}\text { Pro- } \\
\text { teus } \\
\text { spp. }\end{array}$ & $\begin{array}{c}\text { P } \\
\text { value }\end{array}$ \\
\hline $\begin{array}{l}\text { No } \\
\text { mps }\end{array}$ & 159 & $63(39.6)$ & $40(25.2)$ & $(6.9)$ & 0.001 \\
& & & & & \\
$1+$ & 71 & $14(19.7)$ & $12(16.9)$ & $4(5.6)$ & \\
$2+$ & 39 & $7(17.9)$ & $5(12.8)$ & $0(0.0)$ & \\
$3+$ & 13 & $2(15.4)$ & $1(7.7)$ & $0(0.0)$ & \\
$4+$ & 2 & $0(0.0)$ & $0(0.0)$ & $0(0.0)$ & \\
\hline
\end{tabular}


Malaria and UTI among children

Ephraim et al.,

very heavy parasite density (4+), no bacteria was isolated (Table 3).

Demographic characteristics of participants with malaria co-existing with UTI infection is as summarized in Table 4. Age was significantly $(\mathrm{P}=0.025)$ associated with co-infection. Children aged up to 36 months had the highest prevalence of co-infection $(82.2 \%)$. More females (58\%) than males $(42 \%)$ had malaria and UTI co-infection. However, gender was not significantly $(\mathrm{P}=0.272)$ associated with coinfection among participants enrolled in this study. Table 5 shows the antimicrobial susceptibility patterns of urine isolates. All the isolates (Staph aureus, E. coli, Proteus $s p p$ ) were highly sensitive to CIP, GEN and NIT with sensitivities ranging from $89 \%$ to $100 \%$. All the organisms showed resistance to ampicillin (0-9.8\%).

The percentage of participants with no bacteriuria predominated the other classification criteria across all the age groups with the most from 37-48 months $(10.21 \%)$ and the least within the 6-12 months (7.04\%). Staphylococcus aureus was the most isolated organism with similar frequencies in the 25-36 months $(9.51 \%)$ and the $13-24$ months $(9.10 \%)$ age categories. The 6-12 months age group gave the least frequency $(3.52 \%)$ of Staphylococcus aureus infection. Escherichia coli was the second highest isolate. The number of E. coli isolates from the 13-24 and the 25-36 months age group were of the same frequency $(4.93 \%)$; the 6-12 month age group gave the least frequency $(2.46 \%)$ of E coli. Proteus species was generally the least of all the organisms isolated from study participants. The frequency of Proteus spp isolated was higher among children aged 13-24 months
Table 4: Prevalence of co-existing urinary tract infection and malaria infection

\begin{tabular}{lccc}
\hline Variable & $\begin{array}{c}\text { Present } \\
\mathbf{s}\end{array}$ & $\begin{array}{c}\text { Absent } \\
(\mathbf{n}=\mathbf{4 5})\end{array}$ & P value \\
\hline Age range & (months) & & \\
$\leq 12$ & $6(13.3)$ & $15(18.8)$ & 0.025 \\
$13-24$ & $16(35.6)$ & $12(15.0)$ & \\
$25-36$ & $15(33.3)$ & $24(30.0)$ & \\
$37-48$ & $5(11.1)$ & $19(24.0)$ & \\
$49-60$ & $3(6.7)$ & $10(12.5)$ & \\
Gender & & & \\
Male & $19(42.0)$ & $42(52.5)$ & 0.272 \\
Female & $26(58.0)$ & $38(47.5)$ & \\
\hline
\end{tabular}

$(2.11 \%)$ than those aged 37-48 and 49-60 months (0.35\% each) (Figure 1).

\section{DISCUSSION}

Co-infection of malaria and UTI is not a new phenomenon. There is generally under-reporting and underestimation of these conditions in children especially as fever, a symptom, is common in both infections (White, 1989; Musa-Aisien et al., 2003; Okunola et al., 2012). This study determined the prevalence of coexisting malaria with UTI, identified the predominant causative agent and also assessed the antimicrobial susceptibility pattern of the isolated organisms among children $<5$ years with fever in the Assin South Municipality of Ghana. The prevalence of malaria and UTI co-infection in this population was $15.8 \%$ with Staphylococcus aureus as the predominant isolate. The isolates were sensitive to ciprofloxacin, nitrofurantoin and gentamicin and resistant to ampicillin.

Table 5: Antimicrobial sensitivity pattern of bacteria isolate

\begin{tabular}{llllllllllll}
\hline $\begin{array}{l}\text { Iso- } \\
\text { lates }\end{array}$ & No. & CIP & GEN & NIT & NAL & CRX & AMP & TET & COT & PPA & CTX \\
\hline Staph & & & & & & & & & & & \\
aureus & 86 & $77(89.5)$ & $82(95.3)$ & $80(93.0)$ & $45(52.3)$ & $55(64.0)$ & $8(9.3)$ & $28(32.5)$ & $25(29.1)$ & $37(43.0)$ & $67(77.9)$ \\
E coli & 58 & $48(82.7)$ & $54(93.1)$ & $56(96.5)$ & $26(44.8)$ & $38(65.5)$ & $5(8.6)$ & $27(46.5)$ & $14(24.1)$ & $28(48.2)$ & $45(77.6)$ \\
Proteus & & & & & & & & & & & \\
species & 15 & $13(86.6)$ & $15(100)$ & $13(86.6)$ & $5(33.3)$ & $11(73.3)$ & $0(0.0)$ & $3(20.0)$ & $6(40.0)$ & $8(53.3)$ & $10(66.6)$ \\
Total & 159 & $13987.4)$ & $151(95.0)$ & $149(93.7)$ & $76(47.8)$ & $104(65.4)$ & $13(8.2)$ & $58(36.4)$ & $45(28.3)$ & $74(46.5)$ & $122(76.7)$ \\
\hline
\end{tabular}

CIP: Ciprofloxacin; GEN: Gentamicin; NIT: Nitrofurantoin; NAL: Nalidixic Acid; CRX: Cefuroxime; AMP: Ampicillin; Tet: Tetracycline; Cot: Cotrimoxazole; PPA: Pipedemic acid; CTX: Ceftriaxone. 
Malaria and UTI among children

Ephraim et al.,

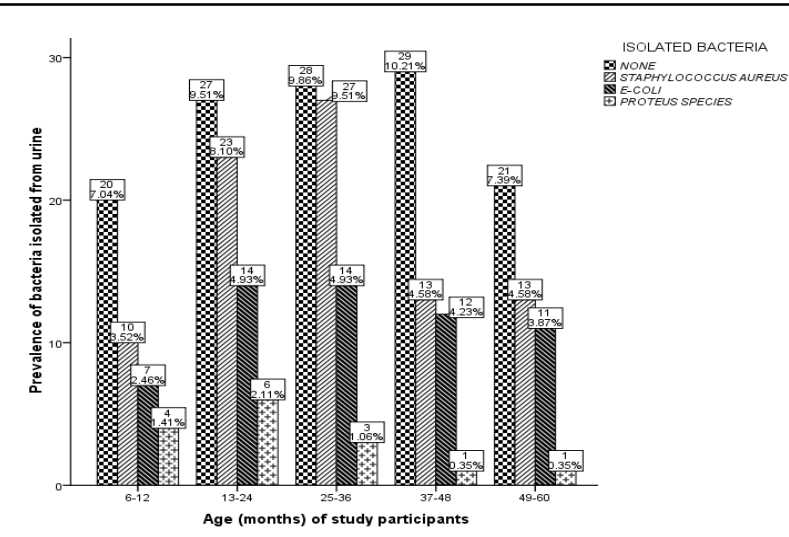

Figure 1: Prevalence of bacteria isolate stratified by age

Co-infection of malaria and UTI in children has been reported by several studies conducted across the African continent (Musa-Aisien et al., 2003; Okwara et al., 2004; Okunola et al., 2012). The prevalence of co-infection reported in this study is a little higher than the $13.3 \%$ recorded in earlier studies in Nigeria (Okunola et al., 2012). It is unclear why variations exist in the prevalence rates among studies which employed similar designs and recruited comparable age groups. However, the use of anaemia in defining the severity of malaria in the Nigerian study could account for this difference.

An earlier study by Okunola et al., (2012) reported a high prevalence of bacteriuria among children aged 13-24 months with malaria, a figure similar to what is recorded in this study though the association between age and bacteriuria was insignificant. Children below the age of twelve months received proper nursing care from their parents especially through breast feeding. However, between 13 months and 48 months children, due to their developing immune system, are exposed to all manner of infectious agents which makes them susceptible to common infections including UTI.

The current study, in contrast to earlier studies (Akpede and Skyes, 1992; Okwara et al., 2004) observed a significant association between parasite density and bacteriuria. This could be attributed to the high prevalence of malaria, and the relatively younger age of participants recruited in this study.
Data on the predominant isolate from UTI's in children has been inconsistent. Earlier works by Osegbe et al.,(1991), Musa-Asien et al., (2003) and Okwara et al., (2004) reported E. coli as the predominant isolate. However, in agreement with the work of Okunola et al., (2012) Staphylococcus aureus was identified as the main isolate among children in the Assin South Municipality. The predominance of Staphylococcus aureus in this study compared to earlier studies could mean that children with malaria are more susceptible to infections with gram positive organisms. In consonance with an earlier study (Okunola et al., 2012) only three pathogens were isolated from our participants. These could be the only pathogens involved in the infection or other less common organisms or organisms with special nutritional and growth requirements could be present but the use of routine media made it impossible for us to isolate and identify these organisms.

On antimicrobial sensitivity testing, all the isolates were highly sensitive to gentamicin, ciprofloxacin and nitrofurantoin but less sensitive to ampicillin. This observation is consistent with the findings of earlier studies (Okunola et al., 2012) which described the emergence of uropathogens resistant to commonly used antimicrobials. The high incidence of resistance of the isolated pathogens to ampicillin may be due to the indiscriminate use of the drug. However, it must noted that the challenges associated with the use of these antibiotics such as having to inject it parenterally as in the case of gentamicin, and the numerous side effects of nitrofurantoin and ciprofloxacin might have reduced its usage among patients.

\section{CONCLUSION}

Co-infection of malaria and UTI was present in $15.8 \%$ of febrile children $<5$ in the Assin South municipality. Staphylococcus aureus was the predominant cause of the UTI and the isolates were highly resistant to ampicillin but susceptible to gentamicin, ciprofloxacin and nitrofurantoin. Health care personnel should not rule out UTI when managing febrile children $<5$ years with malaria. 
Malaria and UTI among children

Ephraim et al.,

\section{ACKNOWLEDGEMENTS}

The authors are grateful to the participants and the staff of Saint Francis Xavier Hospital, in the Assin Central Municipality of the Central Region and also staff of Laboratory Technology department, University of Cape Coast, Ghana.

\section{COMPETING INTERESTS}

The authors declare that they have no competing interests.

\section{REFERENCES}

Akpede G.O. and Skyes R.M. (1992) Relative contribution of bacteremia and malaria to acute fever without localizing signs of infections in under five children. J Trop Pediatr 38, 295-298.

Bauer A.W., M. Kirby, J. C. Sherris and Turck. M. (1966) Antibiotic susceptibility testing by a standardized single disk method. Am. J. Clin. Pathol 45:, 493-494.

English M., Reyburn H., Goodman C. and Snow R.W. (2009) Abandoning presumptive antimalarial treatment for febrile children aged less than five years-a case of running before we can walk? PLoS Med 6, e1000015.

Gething P.W., Kirui V.C., Alegana V.A., Okiro E.A., Noor A.M. and Snow R.W. (2010) Estimating the number of paediatric fevers associated with malaria infection presenting to Africa's public health sector in 2007. PLoS Med 7, e1000301.

Harding G.K., Zhanel G.G., Nicolle L.E. and Cheang M. (2002) Antimicrobial treatment in diabetic women with asymptomatic bacteriuria. N Engl J Med 347, 1576-1583.

Kallander K., Nsungwa-Sabiiti J. and Peterson S. (2004) Symptom overlap for malaria and pneumonia-policy implications for home management strategies. Acta Trop 90, 211-214.

Musa-Aisien A., Ibadin M., Ukoh G. and Akpede G.
(2003) Prevalence and antimicrobial sensitivity pattern in UTI in febrile under 5 s in children emergency unit Nigeria. Ann Trop Paediatr 23, 3945.

Okunola P., Ibadin M., Ofovwe G. and Ukoh G. (2012) Coexistence of UTI and malaria among children under five years old. A report from Benin City, Nigeria. Saudi J Kidney Dis Transpl 23(3), 629-634.

Okwara F.N., Obimbo E.M., Wafula E.M. and Murila F.V. (2004) Bacteremia, urinary tract infection and malaria in hospitalized febrile children in Nairobi: is there an association. East African Medical Journal 81, 47-51.

Osegbe D., Adesanya A., Bode C. and Anyiwo C. (1991) Informed choice of antimicrobial agent on urinary tract infection. Nig J Surg Sci 1.

Reyburn H., Ruanda J., Mwerinde O. and Drakeley C. (2006) The contribution of microscopy to targeting antimalarial treatment in a low transmission area of Tanzania. Malar J 5, 4.

Rougemont A., Breslow N., Brenner E., Moret A.L., Dumbo O., Dolo A., Soula G. and Perrin L. (1991) Epidemiological basis for clinical diagnosis of childhood malaria in endemic zone in West Africa. Lancet 338, 1292-1295.

Tagoe D.N.A. and Desbordes K.K. (2012) Investigating potential sources of transmission of healthcare associated infections in a regional hospital, Ghana. Int J App Basic Med Res 2, 20-24.

White R.H. (1989) Vesicoureteric reflux and renal scarring. Arch Dis Child 64, 407-412.

WHO (1991a) Basic laboratory methods in medical parasitology. Special techniques for plasmodia. In WHO pp. pp 48. Geneva.

WHO (1991b) Basic malaria microscopy: Part I Learner' s guide; Part II tutor's guide. Geneva: World Health Organization.

WHO (2000) Communicable Diseases Cluster: Severe falciparum malaria. Trans R Soc Trop Med Hyg 94 (Suppl 1), S1-90.
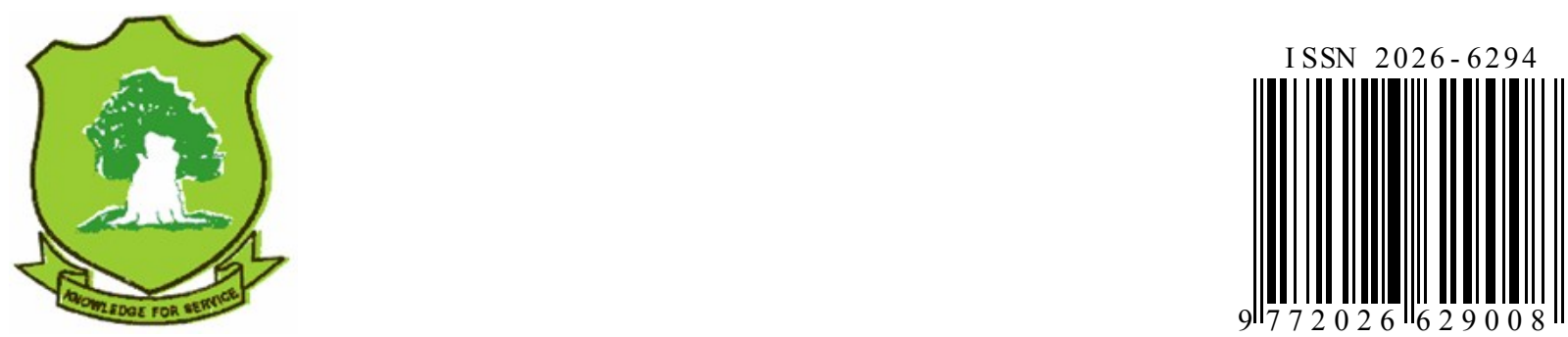